\title{
Multi-agent control system with intelligent optimisation for building energy and comfort management
}

\section{Ee May Kan* and Siew Leong Kan}

School of Engineering,

Nanyang Polytechnic,

180 Ang Mo Kio Avenue 8, 569830, Singapore

Email: kan_ee_may@nyp.edu.sg

Email: kan_siew_leong@nyp.edu.sg

*Corresponding author

\section{Yvonne Soh}

Singapore Green Building Council, 390 Havelock Road \#06-05,

King's Centre, 169662, Singapore

Email: Yvonne_soh@sgbc.sg

\section{Dou Zi}

School of Engineering,

Nanyang Polytechnic,

180 Ang Mo Kio Avenue 8, 569830, Singapore

Email: dou_zi@nyp.edu.sg

\section{Khaing Yadanar}

School of Electrical and Electronic Engineering,

Nanyang Technological University,

50 Nanyang Ave, 639798, Singapore

Email: khaing004@e.ntu.edu.sg

\begin{abstract}
Smart, sustainable and energy-efficient buildings have recently become a trend for intelligent and green building industry. One of the challenges for such a building is to minimise power consumption without compromising the occupant comfort. It is possible to sustain high comfort level with minimal energy consumption through intelligent multi-agent control and optimisation. Our work focuses on a new algorithmic aspect that makes buildings more intelligent, resource efficient and comfortable for occupants. A multi-agent control system with intelligent optimisation is proposed to optimise heating, ventilation and air-conditioning (HVAC) processes using computational intelligence approaches. The optimum solutions generated through the optimisation engine and task scheduling for each agent will be used to sustain a high level of occupant comfort with minimal power consumption, increase energy utilisation efficiency, and consequently reduce energy cost.
\end{abstract}


Experimental results and comparisons with other evolutionary approaches demonstrate the overall performance and potential benefits of the new framework.

Keywords: multi-agent control; optimisation; computational intelligence.

Reference to this paper should be made as follows: Kan, E.M., Kan, S.L., Soh, Y., Zi, D. and Yadanar, K. (2016) 'Multi-agent control system with intelligent optimisation for building energy and comfort management', Int. J. Automation and Logistics, Vol. 2, Nos. 1/2, pp.60-77.

Biographical notes: Ee May Kan received her Bachelor and PhD degrees in Electrical and Electronic Engineering from Nanyang Technological University (NTU), Singapore. Currently, she is a Principal Investigator of the 4th MOE-TIF research grant. She supervises students in industry collaboration projects and competitions. Her research interests include intelligent systems, evolutionary computation, computational intelligence and control theory.

Siew Leong Kan is currently a Manager of the Communications and Networks Group in Nanyang Polytechnic. He received his BSc in Communications Engineering (first class hons.) from the University of Kent at Canterbury, and MSc in Communications and Computer Networks from the NTU. His current research interests include sensor networks, and Internet of Things. His work has resulted in two US granted patents, seven Singapore granted patents, and one patent pending. His work on secured MANET routing engine, which was awarded the 4th NRF-POC grant in 2010, has attracted a company signing an Intellectual Property (IP) Licensing Agreement with the Polytechnic.

Yvonne Soh is a Professional Engineer and currently working as an Executive Director at Singapore Green Building Council. She graduated with a Bachelor of Engineering (Civil) from NTU and obtained her Master of Science (Civil Engineering) degree from NUS. She gained her industry experience as a civil engineer at P\&T Consultants, an international multi-disciplinary building and construction consultancy firm, before joining BCA.

Dou $\mathrm{Zi}$ is an Electrical and Electronic Engineering (EEE) student at Nanyang Technological University. She received her diploma with merit in Multimedia and Infocomm Technology from Nanyang Polytechnic, Singapore. She had won the Tan Tah Kee Young Investor's Commendation Award 2015 in Open Category.

Khaing Yadanar is an Electrical Engineer currently working in CPG Consultants Pte Ltd. She obtained first class honours degree in Electrical and Electronic Engineering from Nanyang Technological University. She obtained a diploma with merit in Electronic and Computer Engineering from Ngee Ann Polytechnic with two diploma plus certificates in Microelectronic and Robotic. During Singapore Robotic Games 2013, her team was the second runner up for the Robot Colony Competition. In addition, she obtained the first of overall best performance in Level 1 semester 1 ECE course in Polytechnic.

This paper is a revised and expanded version of a paper entitled 'Multi-agent control system with intelligent optimization for building energy management' presented at the 18th Asia Pacific Symposium of Intelligent and Evolutionary Systems, Singapore, 10-12 November 2014. 


\section{Introduction}

Strategies towards sustainable performance with occupants have become increasingly important. Optimal indoor conditions help to enhance occupant health, productivity and experience. There have been multiple goals and provisions for the improvement of occupant comfort and energy savings (Dounis and Caraiscos, 2009; Fong et al., 2009; Kolokotsa et al., 2002). The improvement of the indoor environment comfort demands more energy consumption and the building operations require high energy efficiency to reduce energy consumption. Thus, one of the most important issues on energy-efficient buildings is to optimise the requirements of the occupants' comfort and power consumption effectively.

A multi-objective genetic algorithm (MOGA) is employed in Pervez et al. (2013) to study the behavioural model relationship between energy consumption and thermal comfort. Multi-island generic algorithm (MIGA) and generic algorithm (GA) is utilised in Ali and Kim (2013) to build the model for balancing the energy consumption and occupant comfort level. An overview of population-based optimisation techniques and the methodologies used for multiple objective problems is discussed in Giagkiozis et al. (2013). It is possible to sustain high comfort level with minimal energy consumption through intelligent control of building energy management systems. Intelligent control of the thermal comfort, visual comfort and air quality comfort are vital for both energy efficiency and occupant's quality of living (Dounis and Caraiscos, 2009). These three basic factors determine the occupants' quality of lives in a building environment. Thus, the basic control objective for the multi-agent control system is to sustain the occupant comfort level while minimising the energy footprint of buildings. A framework for addressing building energy management with objectives of maximising occupant comfort, increased energy efficiency, decreased operation cost of energy utilisation, and consequently decreased greenhouse gas emissions is used to set as an optimisation goal for the current work. The work described will deal with an application of multi-agent coordination control for building management via data fusion and analysis for real-time monitoring and control of energy distributions in buildings and cyber integration along the lines of contemporary mandates.

Our focus in this work is on computational intelligence approach to optimise multi-objective heating, ventilation and air-conditioning (HVAC) processes. There are several important components considered in the proposed approach, for instance, the representation of the multi-objective optimisation problem, the fitness function as well as the genetic search operators. With proper and domain-specific representation, we can easily apply our proposed approach to the multi-objective optimisation problem. We take into consideration the building operation constraints while optimising the HVAC processes. The rest of this paper is organised as follows. Details on the formulation of the problem are presented in Section 2. Section 3 and Section 4 present the solution and implementation, respectively, of the proposed approach substantiated with simulation results. Section 5 shows the results compared with the existing evolutionary approach with brief discussions. Lastly, we summarise the main contributions of this study in Section 6, and enlist several recommendations for the future research. 


\section{Problem formulation}

The multi-objective optimisation problem for building energy management can be formulated in terms of an energy cost function subject to constraints of the building operations. We consider the multi-objective problem as an optimisation problem with the conflicting objectives of minimising the power consumption, maximising the air quality comfort and maximising the thermal comfort. Intelligent control of the thermal comfort and air quality comfort are vital for both energy efficiency and occupant's quality of living. The multi-objective optimisation problem is defined as follows:

$$
\begin{aligned}
& F=\sum_{i=1}^{n} w(i) f(i) \\
& f(i)=\delta_{1}\left[1-\left(\frac{e_{T}}{T_{\text {set }}}\right)^{2}\right]+\delta_{2}\left[1-\left(\frac{e_{L}}{L_{\text {set }}}\right)^{2}\right]+\delta_{3}\left[1-\left(\frac{e_{A}}{A_{\text {set }}}\right)^{2}\right], \quad 1 \leq i \leq n
\end{aligned}
$$

where $F$ indicates the overall occupant comfort level for multiple zones, $w$ is the weighting coefficient for zone $i, f(i)$ represents the overall customer comfort level, which falls into $[0,1]$. It is the control goal to be maximised; $\delta_{1}, \delta_{2}$ and $\delta_{3}$ are the users-defined factors, which indicate the importance of comfort factors. $\delta_{1}, \delta_{2}$ and $\delta_{3}$ fall into [0,1], and $\delta_{1}+\delta_{2}+\delta_{3}=1$. $e$ is the difference between set point and actual sensor measurement; $T_{\text {set }}$, $L_{\text {set }}$ and $A_{\text {set }}$ are the set points of temperature, illumination and air quality, respectively. The goal of the optimisation algorithm is to identify the optimal set of solutions. However, identifying the entire optimal set is practically impossible due to its size. In addition, for many problems, especially for combinatorial optimisation problems, proof of solution optimality is computationally infeasible. Therefore, a practical approach to combinatorial optimisation is to investigate a set of solutions that represent the optimal set within a given computational time limit. The optimisation engine generates smart solutions that can sustain a high level comfort (based on the user's requirements) with minimal energy consumption.

\section{Solution methodology}

\subsection{Multi-objective optimisation for building energy and comfort management}

The technology of multi-agent control system has been successfully utilised in various engineering fields. The fundamental element in multi-agent control system is the agent, which can be software or physical entity. In the current work, a hierarchical multiple agents-based control system is designed for building energy and human comfort management in the smart building. Figure 1 shows the overall system architecture. Based on the customer preferences and the set points predefined by the users, the multi-agent control system is utilised to reduce the error between the set points and sensor measured values so that the high comfort level is maintained and the energy consumption is reduced. The multi-agent control system is classified into different zone agents based on the distinct functions at multiple zones. The zone agents monitor the energy flow and responsible for energy management in its specific zone based on the occupant preference. The proposed algorithm is embedded in the master agent to optimise the set points. 
Multiple control agents are used to control the devices which are related to the comfort factors. The main comfort factors considered in this study include environmental temperature, illumination level and indoor air quality. Accordingly, the control agents are classified into the temperature control agent, the illumination control agent, and the air-quality control agent. Through the cooperation of these multiple agents, the overall goal can be realised.

Figure 1 Framework of the multi-agent control and management system (see online version for colours)

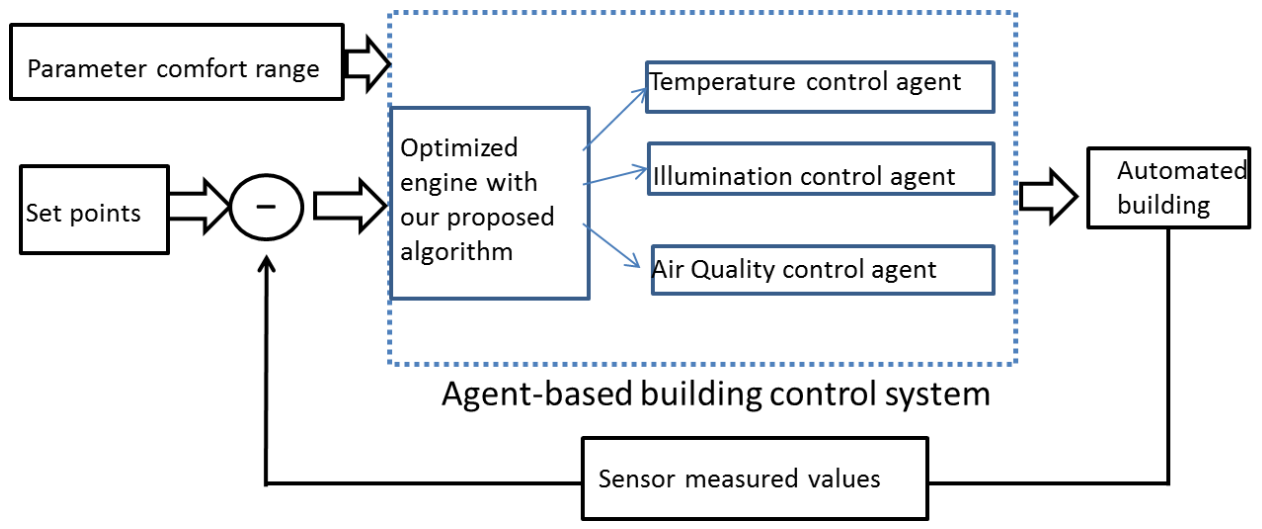

In the current work, a hierarchical multiple agents-based control system is designed for building energy and human comfort management in the smart building. The proposed framework consists of two elements which are server control engine (energy management system) and client control application (cloud server and mobile application). Users can access energy management system through cloud-based server with remote control functionality. The proposed framework consists of three parts: energy management designer, energy management optimiser and energy management comparer. Firstly, energy management designer allows users to design and customise their floor layout in the simulator; next, energy management optimiser made use of the proposed algorithm (as shown in Figure 2) to generate the smart energy solutions in the form of task scheduling for multiple agents which can maintain HVAC in a higher occupant comfort level and energy efficiency than initial scheduling. Finally, energy management comparer provides the decision-making options that include the trade-offs by the comparisons of sensor values. Based on the occupant preferences and the set points predefined by the users, the multi-agent control system is utilised to reduce the error between the set points and sensor measured values so that the high comfort level is maintained and the energy consumption is reduced. The multi-agent control system is classified into different zone agents based on the distinct functions at multiple zones. The zone agents monitor the energy flow and responsible for energy management in its specific zone based on the occupant preference. The proposed algorithm is embedded in the master agent to optimise the set points. Multiple control agents are used to control the devices which are related to the comfort factors. The main comfort factors considered in this study include environmental temperature, illumination level and indoor air quality. Accordingly, the control agents are classified into the temperature control agent, the illumination control 
agent, and the air-quality control agent. Through the cooperation of these multiple agents, the overall goal can be realised.

In this study, the proposed algorithm is used to tune the set points according to the customer preference. As different users have different preferences, a GUI simulation platform has been developed, which offers the flexibility to customers to set their different comfort levels $\left[T_{\min }, T_{\max }\right],\left[L_{\min }, L_{\max }\right]$ and $\left[A_{\min }, A_{\max }\right]$ for temperature, illumination, and humidity, respectively. The three comfort zones predefined by the occupants according to their preferences will generate a three-dimensional restriction area to the optimisation problem. During the initialisation of the particles, the initial location should be within these users-defined zones. The objective function is defined in (1) and (2), so the optimisation goal is to maximise the objective function within the search space. The particle flies within the restriction space according to its memory and moves towards its best local position $L_{\text {best }}$. When the termination condition is met, the particle with the highest fitness value is returned which contains the sequence of set points for each zone. This sequence of set points represents the solutions for power distribution and the corresponding overall comfort.

Figure 2 Generic framework of the proposed algorithm (see online version for colours)

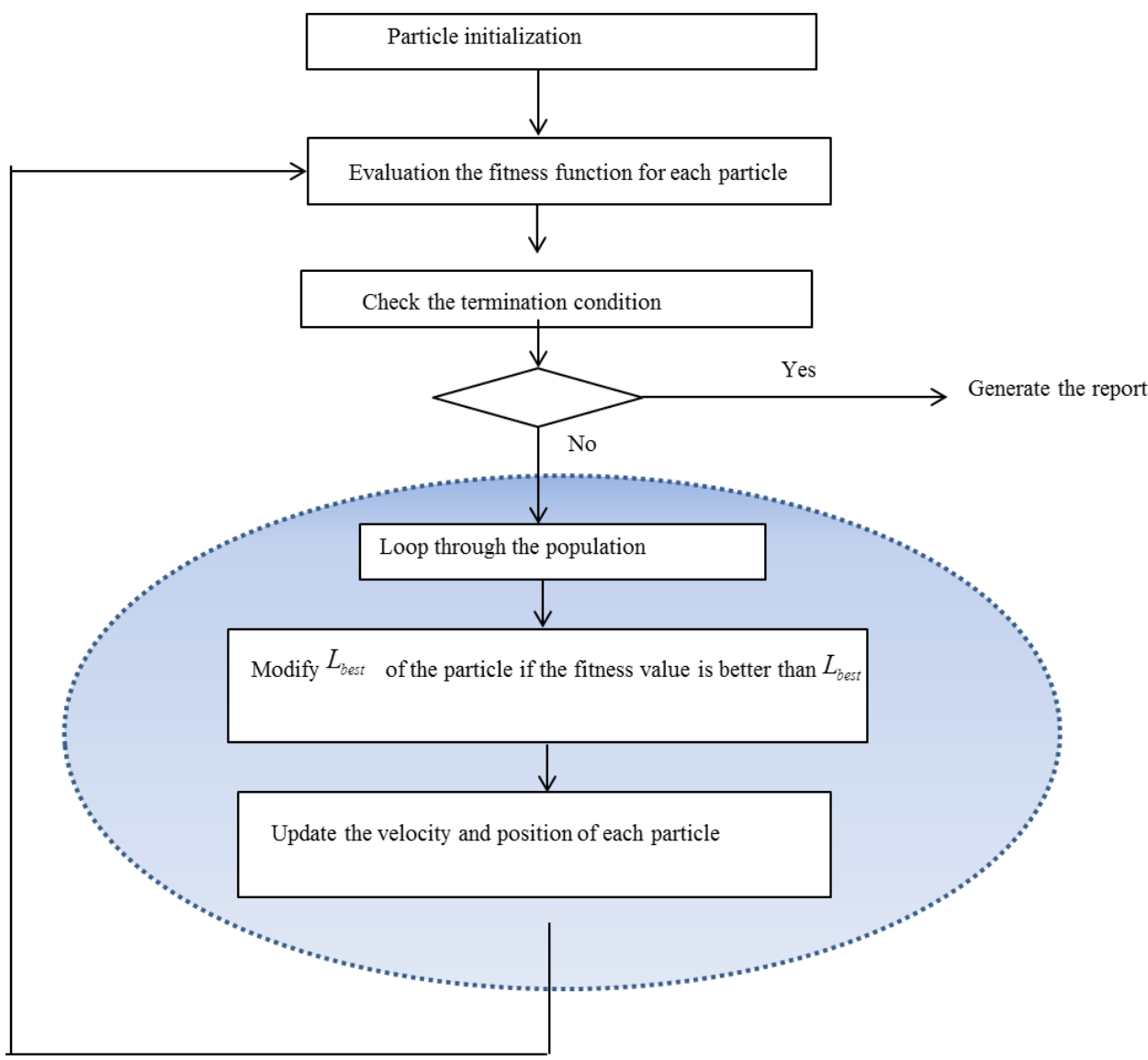




\subsection{The proposed algorithm}

The proposed algorithm involves initialisation, evaluation and exploration of population. A single local search having certain learning roles and search features, would work effectively on a given problem of certain properties but fail to improve solutions on problems that possess contradictory properties. Hence, we propose a cooperative individual learning procedure so that multiple local search are hybridised and organised in a cooperative manner; they can work together to accomplish their shared optimisation goal, thus making the proposed algorithm robust and effective. We describe the proposed individual learning procedure as three distinct cooperative strategies, each having unique learning roles and search features. The three strategies of the individual learning procedure are described in greater details in the following sections. In our proposed algorithm, we first evaluate the particles (potential solutions) and check for dominance relation among the population. Next, we search for non-dominated solutions and store them as potential candidates in order to lead the search particles. It is worth noting that the variable size of the sequence of potential candidates is to improve the computational efficiency of the algorithm during optimisation. Additionally, we make use of crowding distance assignment operator to trim down the size of the candidates if it exceeds the allowable size. Lastly, we exploit an efficient evaluation strategy for evaluating the search space. By combining these operators, the algorithm is competent to sustain diversity in the population and successfully explore towards true Pareto optimal fronts. The main steps of the procedure are described as follow.

\subsubsection{Sequence of potential candidates}

The performance evaluation and selection of the best global particle guide are vital procedures in a multi-objective optimisation approach. They sustain a superior range of non-dominated solutions and influence the convergence capability of the algorithm. In the preceding iteration of the procedure, any of the non-dominated solutions from the sequence of potential candidates can be chosen as a global guide. We propose a performance evaluation method to make sure every particle in the population moves towards the true Pareto optimal region. Moreover, the crowding distance operator is used to perform restriction on the waypoint candidates. This operator ensures the highest crowding distance values of non-dominated solutions are stored as potential candidates. Subsequently, we evaluate and select the best global particle guide from a restricted size of the potential candidates. During optimisation, the variable size of the potential candidates manages to reduce the computational time. However, the computing requirement for the sorting and crowding value becomes greater when the size increases. Hence in the initial steps of the procedure, we set the size to half of the maximum size of potential candidates; followed by increasing the value in a stepwise manner to explore the solution space effectively. This procedure evaluates distinct particle guides from a restricted number of potential candidates and thus improves the algorithm performance by enabling the particles explore towards the true Pareto optimal region. 


\subsubsection{Crowding distance assignment operator}

The crowding distance assignment operator specifies an estimate of the density of solutions. In a particular solution, the average distance of two neighbouring solutions is the crowding distance value. We compute the crowding distance value by first sorting the solution sequence in ascending order of the objective function values and followed by securing boundary solutions with the smallest and greatest values of the objective function. Subsequently, the final crowding distance value of a solution is computed by adding up the individual crowding distance values in every objective function. The detailed computation of crowding distance value is presented as follows:

1 Obtain the size of non-dominated solutions from the sequence of potential candidates

$$
l=|S| \text { where } S=\left[W_{1}, W_{2}, \cdots, W_{k}\right]
$$

2 Initialise distance.

$$
\begin{aligned}
& \text { For } i=1 \text { to } l \\
& S[i] . d i s t=0
\end{aligned}
$$

3 Calculate the individual crowding distance value.

4 Sort each solution according to objective function value.

For each objective $m$

$$
S=\operatorname{sort}(S, m)
$$

5 Set the boundary points to infinite to guarantee their selection.

$$
\begin{aligned}
& S[1] . \text { dist }=S[l] . \text { dist }=\infty \\
& \text { For } i=2 \text { to }(l-1) \\
& S[i] . \text { dist }=S[i] . d i s t+\frac{s[i+1] \cdot m-s[i-1] . m}{f_{m}^{\max }-f_{m}^{\min }}
\end{aligned}
$$

\subsubsection{Generic evaluation operator}

In this work, we apply the generic evaluation operator involving priorities, goals and Pareto sets in which we can place the objectives in levels of priority and impose constraints on each of them. The operator evaluates all non-dominated solutions in the population, determines if one Pareto dominates the other, and ranks them accordingly. In order to set the priority levels of our objectives for the optimisation problem, we classify them as follows:

- constraints that the agent has to fulfil due to its building operations, or user requirements

- zone-specific energy costs to be optimised. 
The constraints that the agent must fulfil are set as high-priority levels. On the other hand, the energy costs to be optimised are set at lower priority levels in line with the goal of the optimisation. By using this method, we can simply modify the constraints or zone-specific energy costs as required by the different group of users. This improves the performance of multi-objective optimisation while extending from conventional optimisation algorithm. The evaluation operator works on a specific number of particles and distributes the non-dominated solutions along the true Pareto optimal front. Initially, this operator strives to substitute the non-feasible solutions with the evaluated particles of potential candidates. Subsequently, it attempts to exploit the search space in the sequence of waypoint candidates along the Pareto fronts. The evaluation procedure is described as follows.

1 Sort the particle fitness function and get the particle index number respectively.

2 Employ crowding distance assignment operator for the calculation of the density of solutions in the sequence of waypoint candidates. Sort them accordingly and choose one of the least crowded solutions from the waypoint candidates randomly as particle guide.

3 Evaluate a specific number of particles and rank them accordingly.

\subsubsection{Constraint handling mechanism}

We adopt the constraint handling mechanism to deal with the constrained optimisation problems. In this simple and effective method, a solution $i$ is considered as a constrained dominate solution $j$ if it meets any of the conditions as follows:

1 Solution $i$ is deemed as feasible while solution $j$ is not.

2 Both solutions $i$ and $j$ are deemed as non-feasible, although solution $i$ has a smaller overall constraint violation.

3 Both solutions $i$ and $j$ are deemed as feasible when solution $i$ dominates solution $j$.

\subsection{The multi-agent control system}

The multi-agent control system is classified into different agents based on the distinct functions at different zones. The zone agents monitor the energy flow and responsible for energy management in its specific zone based on the occupant preference. The proposed algorithm is embedded in the central coordinator-agent to optimise the set points. Multiple local controller-agents are used to control the devices which are related to the comfort factors. The main comfort factors considered in this study include environmental temperature, illumination level and indoor air quality. Accordingly, the local controller-agents are classified into the temperature controller-agent, the illumination controller-agent, and the air-quality controller-agent.

Through the cooperation of these multiple agents, the control objective which is to maximise the occupant comfort and minimise the energy consumption simultaneously can be achieved. By way of example, we consider the multiple building zones with multiple agents portrayed in Figure 3. 
Figure 3 The multiple zones of a building (see online version for colours)

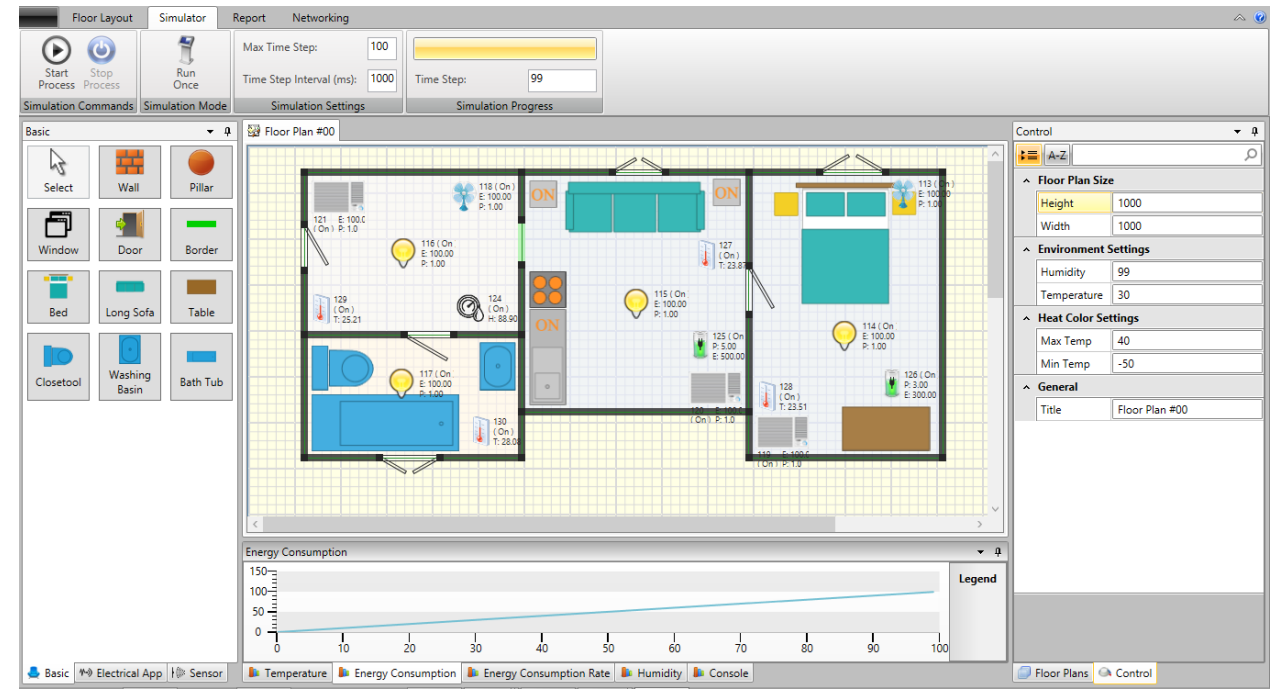

Lastly, we demonstrate the efficiency and applicability of the proposed approach in the following sections.

\section{Experimental results}

In this study, we use set coverage metric (SC) and spacing metric (SP) as performance measures to assess the performance of our proposed approach. Since heuristics algorithms have no guarantee to find the global optimal solution within the limited iterations, in this study we apply our proposed approach by setting the initial population of the algorithm to 200 and specify the size of non-dominated solutions as 200 . We then run the algorithm for 500 iteration steps to increase the possibility of achieving the global optimisation. Generally, more runs of the optimisation algorithm will lead to a higher probability of achieving better results, but it will inevitably require more computational time. After many trials, it was found that 500 is a reasonable number of runs for balancing the solution quality and computational cost. The SC (A, B) metric produces the solution dominance and relative convergence between two solution vectors $\mathrm{A}$ and $\mathrm{B}$; calculating the proportion of $\mathrm{B}$, which is weakly dominated by A. The role of SP metric is to assess the distribution of vectors throughout the set of non-dominated solutions and indicate how evenly the generated non-dominated solutions in the approximation set are distributed in the search space. The computational complexity of the algorithm is dominated by the objective function computation, crowding distance computation and the non-dominated comparison of the particles in the population. If there are $M$ objective functions and $N$ number of solutions (particles) in the population, then the objective function computation has $O(M N)$ computational complexity. For comparison purposes, we run the existing evolutionary algorithm by specifying the initial population as 200; probability of crossover as 0.9 ; and probability of mutation as $1 / n$ ( $n$ is the size of real variables). 
Next, we set the crossover and mutation distribution index values to 20 and 100 , respectively and run the evolutionary algorithm for 500 generations. The main objective is to find an optimum solution which minimises the energy costs while maximising the occupant comfort. The main idea is to have a generated solution with maximal level of occupant comfort while complying with building energy constraints and user requirements. To show the effectiveness of the proposed algorithm, it is applied to energy consumption, air quality comfort and thermal comfort as three objectives of the optimisation engine for solving the multi-objective problem. To examine the performance, we then carry out ten independent runs by applying both the proposed algorithm and the evolutionary algorithm. The resulting statistics for both the algorithms is tabulated in Table 1.

Table 1 Resulting statistics by the proposed algorithm and the existing evolutionary algorithm for the multi-objective problem

\begin{tabular}{|c|c|c|c|c|}
\hline \multirow{3}{*}{ Statistics } & \multicolumn{4}{|c|}{ Performance metric } \\
\hline & \multicolumn{2}{|c|}{ Set coverage (SC) metric } & \multicolumn{2}{|c|}{ Spacing $(S P)$ metric } \\
\hline & $S C(A, B)$ & $S C(B, A)$ & $A$ & $B$ \\
\hline Best & 0.1670 & 0.0360 & 164.9022 & 196.9804 \\
\hline Worst & 0.7031 & 0.5080 & 236.3021 & 630.2212 \\
\hline Mean & 0.4466 & 0.2302 & 206.6202 & 402.4579 \\
\hline Variance & 0.0349 & 0.0254 & $1,136.9908$ & $26,066.7456$ \\
\hline SD & 0.1668 & 0.1426 & 30.1462 & 146.5188 \\
\hline
\end{tabular}

Figure 4 Non-dominated solutions obtained using the proposed algorithm (see online version for colours)

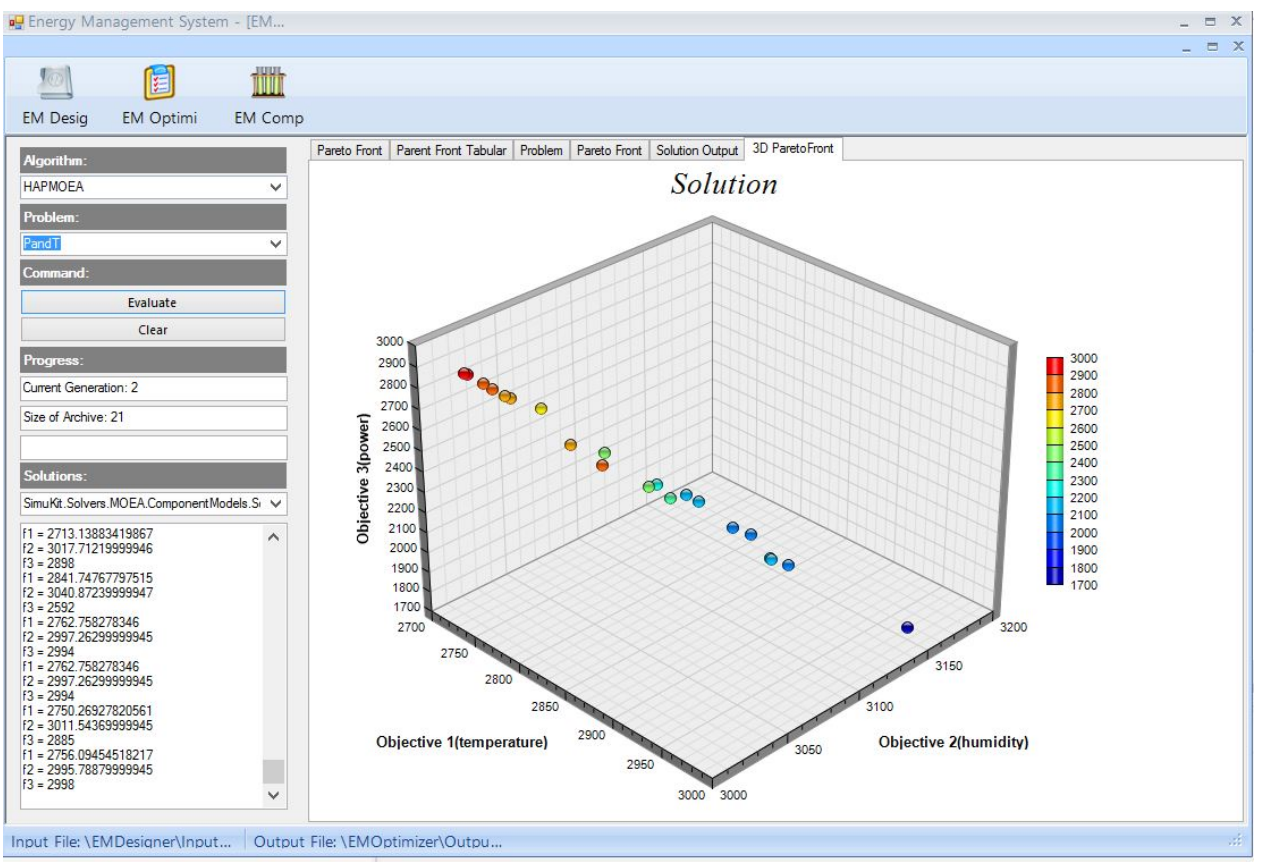


In $\mathrm{SC}(\mathrm{A}, \mathrm{B}), \mathrm{A}$ is the proposed algorithm and $\mathrm{B}$ is the existing evolutionary algorithm. The best performing algorithm is indicated by bold numbers. From Table 1, it can be seen that the mean value of $\mathrm{SC}(\mathrm{A}, \mathrm{B})$ is higher than the mean value of $\mathrm{SC}(\mathrm{B}, \mathrm{A})$ pertaining to set coverage metric. The $\mathrm{SC}(\mathrm{A}, \mathrm{B})$ metric represents the proportion of $\mathrm{B}$ solutions that are weakly dominated by A solutions. Therefore, we can conclude that our proposed algorithm is able to perform better than the existing evolutionary algorithm. Likewise, the proposed algorithm has lower spacing metric mean value than the existing evolutionary algorithm; indicating that the proposed algorithm obtains the better distribution of Pareto optimal solutions. A sample of the experimental result corresponding to $\mathrm{SC}$ (A, B) median value is presented in Figure 4 for illustration purposes.

It can be seen that the proposed algorithm is competent of generating a well-distributed set of Pareto optimal solutions. The remarkable performance of the proposed algorithm can be attributed to the application of the sequence of potential candidates and the generic evaluation operator. The evaluation operator distributes the non-dominated solutions along the true Pareto optimal front and improves the exploratory capabilities of the algorithm to prevent premature convergence. In the preceding iteration of the procedure, any of the non-dominated solutions from the sequence of potential candidates are chosen as a global guide. The selection of the global guide of the particle swarm is a crucial step in a multi-objective algorithm. It affects both the convergence capability of the algorithm as well as the good spread of non-dominated solutions. Our proposed algorithm manages to produce boundary solutions easily in this case.

\subsection{Selection of resultant solution}

It is necessary to reduce the large set of solutions to a few representative solutions after obtaining many solutions which are true Pareto optimal with uniform spread and wide coverage. In order to do that, we make use of a simple clustering algorithm, which reduces the large number of final Pareto solutions $(\mathrm{N})$ to a few representative solutions $(N)$.

\subsection{Clustering approach}

Our proposed approach is capable of finding optimal solutions by trading off the predefined criteria. The procedure is likely to find $N$ non-dominated solutions after running for an adequate number of iterations. However, it is time-consuming to consider only one solution among the $N$ non-dominated solutions. Therefore, we exploit a clustering approach in which only a few representative $N$ non-dominated solutions are well-dispersed from each other on the solution space. Firstly, each solution is assumed to fit into a distinct cluster and the gap between each pair of clusters is then computed. Next, the clusters with the smallest gap are merged together to make up a significant cluster. Subsequently, the procedure is terminated after a satisfactory number of clusters are discovered and only one best solution from each cluster is stored while the rest of them are discarded. The detailed clustering approach is described as follows:

1 Initialise a cluster group $C$; Represent each non-dominated solution $i$ as a separate cluster. i.e., $C_{i}=\{i\}, C=\left\{C_{1}, C_{2}, \ldots, C_{N}\right\}$

2 If, $|C| \leq \bar{N}$ proceed to 5 , else proceed to 3 . 
3 Compute the gap for each pair of clusters by (3),

$$
d_{12}=\frac{1}{\left|c_{1}\right| \cdot\left|c_{2}\right|} \sum_{i \in c_{1}, j \in c_{2}} d(i . j)
$$

where $d_{12}$ denotes the gap between two individuals $i_{1}$ and $i_{2}$ in objective space.

4 Search for $\left(i_{1}, i_{2}\right)$ pair with respect to the smallest gap and followed by merging the two clusters $C_{i_{1}}$ and $C_{i_{2}}$ together. The size of $C$ is reduced by one. Return to \#2.

5 Store the best solution with least average gap from each cluster and discard the rest.

Lastly, with the well-spread clusters available on the solution space, a composite measure is assigned to each cluster based on its location on the space so as to facilitate the selection of resulting optimum solution. The detailed Pseudo-weight vector approach is presented in the following section.

\subsection{Pseudo-weight vector approach}

The pseudo-weight vector is assigned according to the relationship between the found solution and the individual criteria,

$$
\omega_{i}=\frac{\left(f_{i}^{\max }-f_{i}(x)\right) /\left(f_{i}^{\max }-f_{i}^{\min }\right)}{\sum_{m=1}^{M}\left(f_{i}^{\max }-f_{m}(x)\right) /\left(f_{i}^{\max }-f_{m}^{\min }\right)}
$$

where $f_{i}^{\min }$ and $f_{i}^{\max }$ are the minimum and maximum values of the $i^{\text {th }}$ criteria, respectively. Subsequently, a straightforward approach is to select the desired solution with the computed weight vectors. This provides ease in selection of final solution for multi-objective optimisation problem.

\section{$5 \quad$ Results and discussions}

In this section, the optimised solution is going to be compared with the initial layout for the simplest case. The schedules generated from optimisation reduce power consumption. Figure 5 shows the layout simulation before optimisation. The energy consumption can be seen in the right panel. The energy measured for this case is 56 with the initial schedule we set randomly. It can be seen that the temperature is not steady and maintained at the desired temperature which is $26^{\circ} \mathrm{C}$.

The optimised schedule helps to reduce the power consumption while maintaining the desired temperature and humidity. As shown in Figure 6, the energy consumption measured is only 43 using the proposed algorithm. The graph at the bottom of the figure shows that the temperature is maintained at $26^{\circ} \mathrm{C}$.

As mentioned earlier, there are two types of algorithm applied for this multi-objective optimisation process. Using the proposed algorithm, there are 15 solutions generated. User can choose any solution to simulate the optimised zone layout. The optimisation process takes longer with the existing evolutionary algorithm, for e.g., hierarchical asynchronous parallel multi-objective evolutionary algorithm (HAPMOEA). 
Figure 5 Initial layout showing power consumption (see online version for colours)

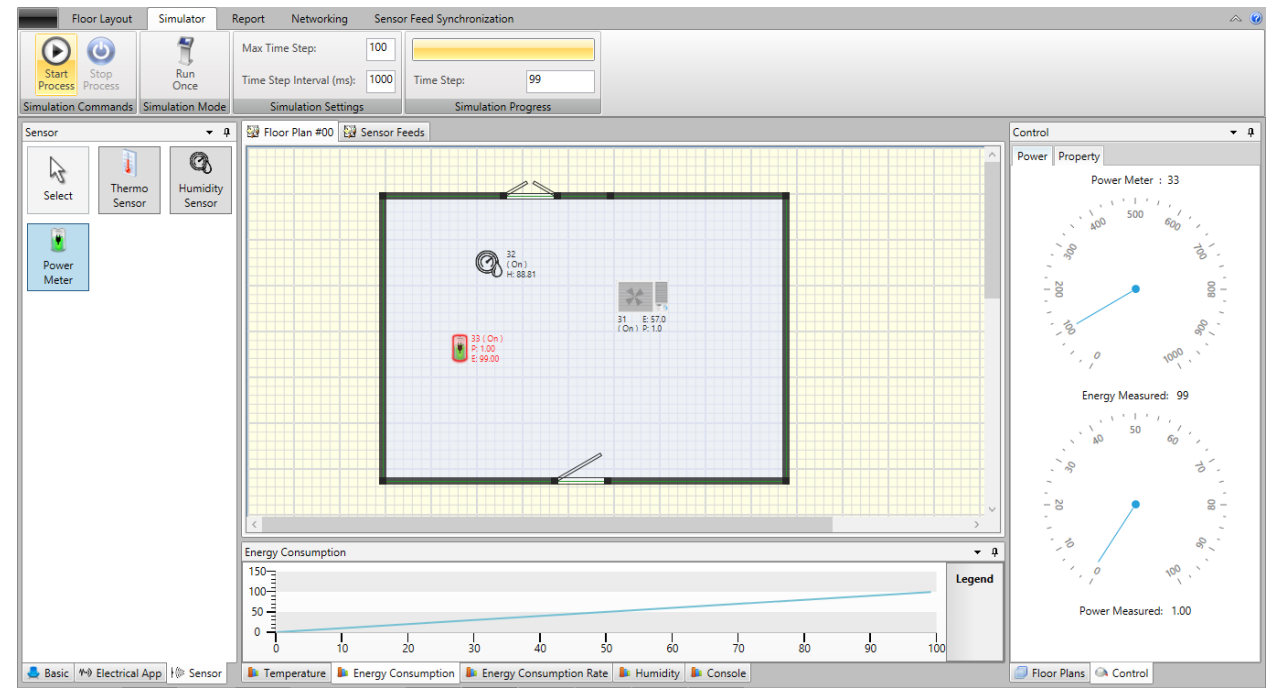

Figure 6 Optimised layout showing power consumption using the proposed algorithm (see online version for colours)

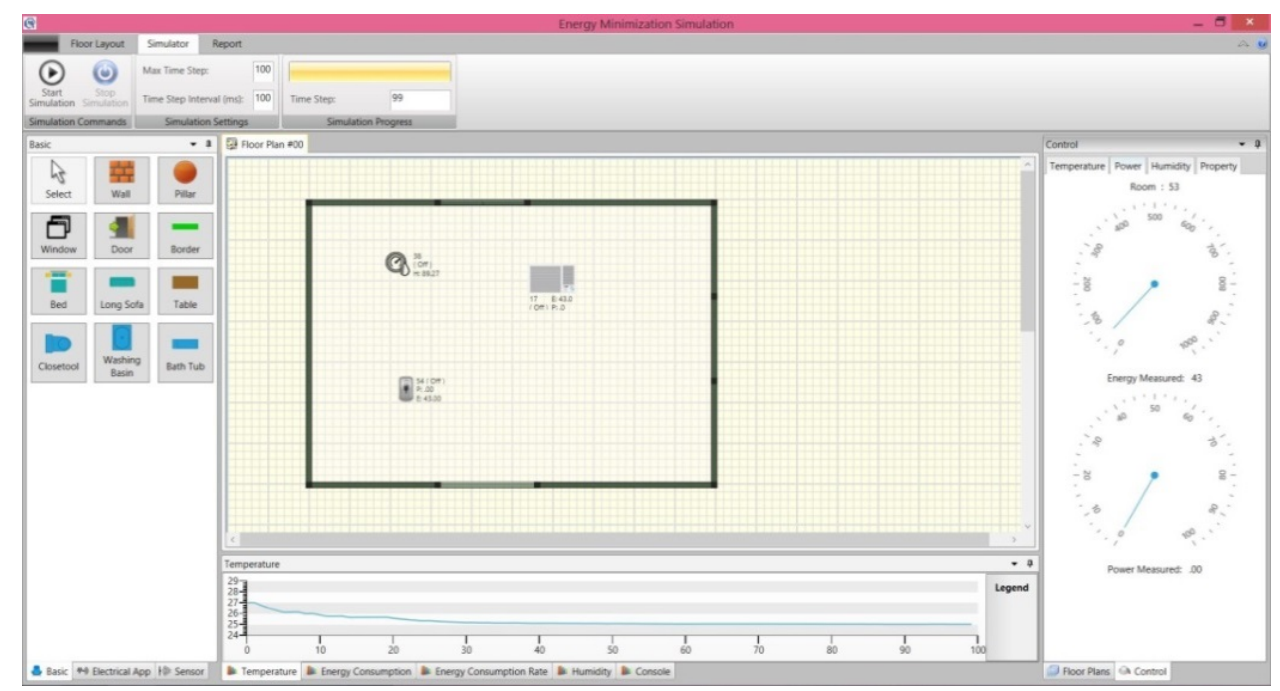

There are 21 solutions generated while the optimisation process is carried out with HAPMOEA and the resulted power consumption is about 48 .

After the optimisation procedure, different solutions will be generated which could be better than the initial schedule or worse than the initial one. But certainly, the optimised schedule will be able to maintain the temperature and humidity at certain comfort level. Next, we conduct the experiments with two zones and two air conditionings. Initially, the total power consumption of the two zones is about 152.8 with unsteady temperature and humidity. 
Figure 7 Optimisation process using HAPMOEA (see online version for colours)

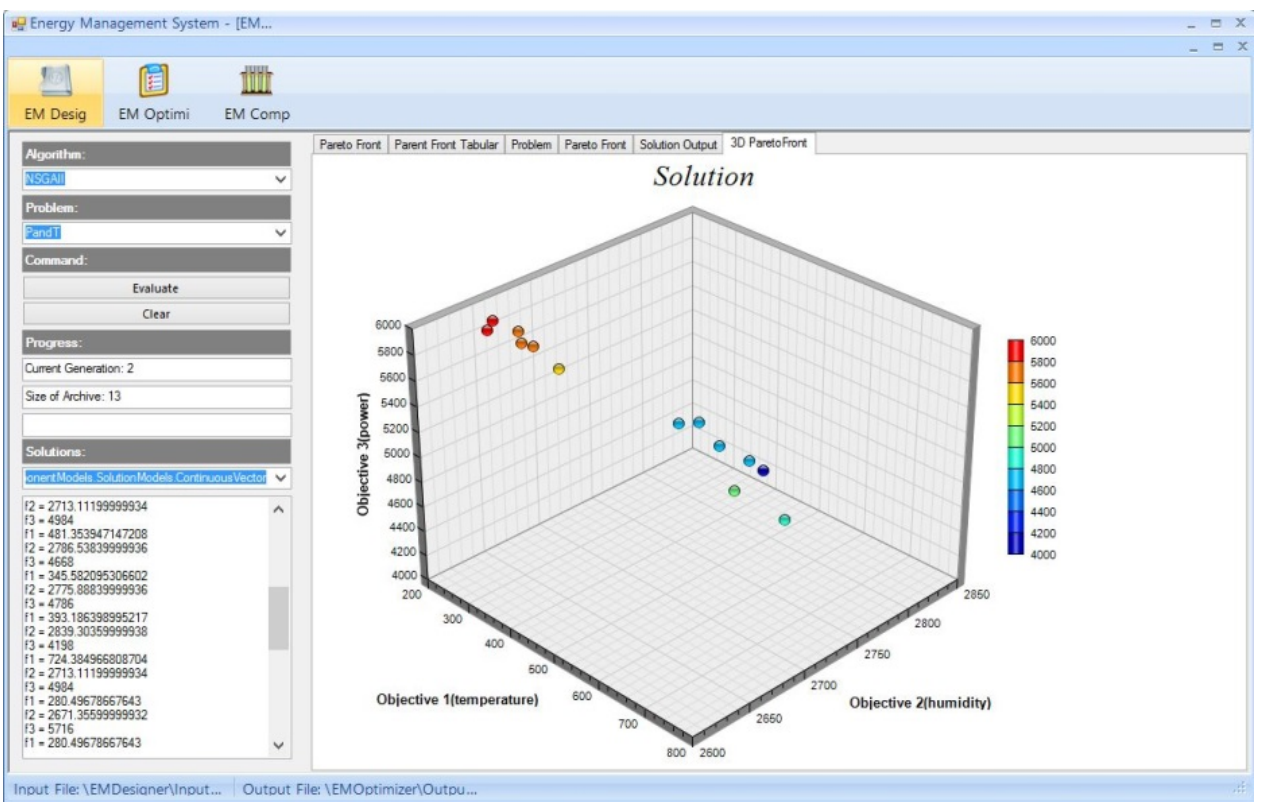

Figure 8 Optimised layout showing power consumption using HAPMOEA (see online version for colours)

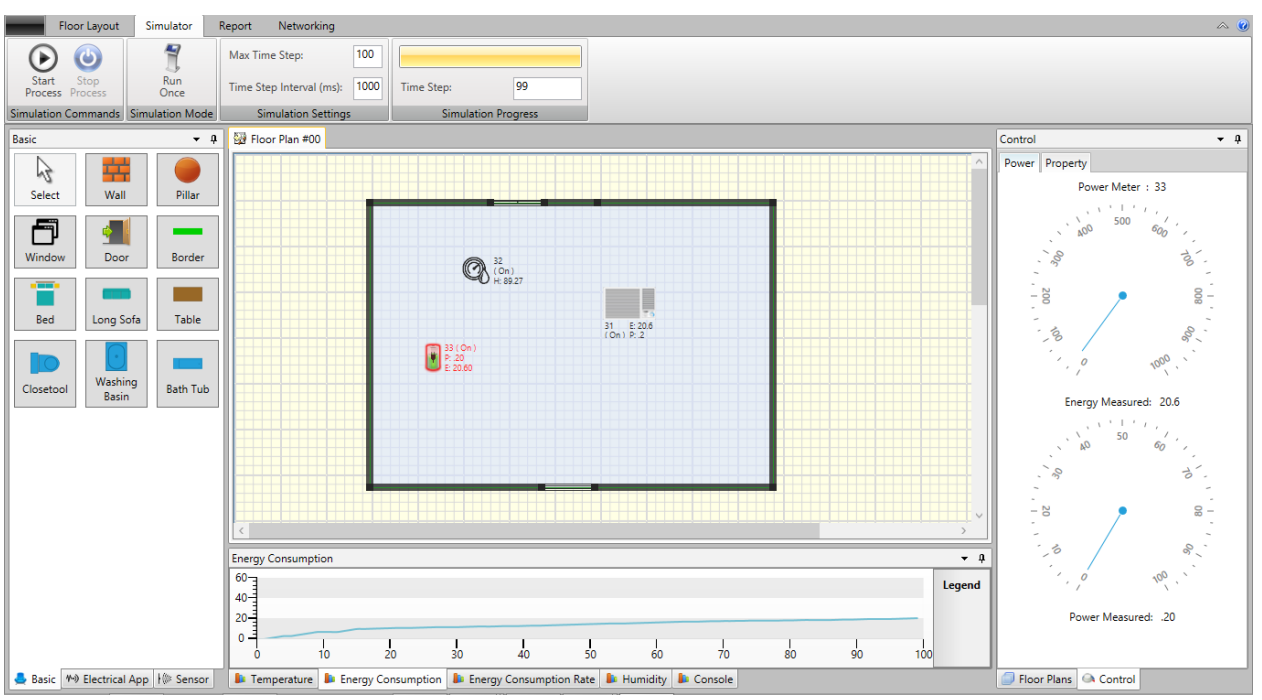

Subsequently, we optimise the schedule with the proposed algorithm. The total power consumption for two rooms is 79.6 while maintaining desired temperature and humidity. 
The optimisation process is then carried out with HAPMOEA. The total power consumption is 82.8 which is higher compared to the solution generated by the proposed algorithm. User can choose the preferred schedule based on their requirement by setting the generation and population size for the optimisation process to have more accurate and desired solutions.

Figure 9 Initial layout showing power consumption (see online version for colours)

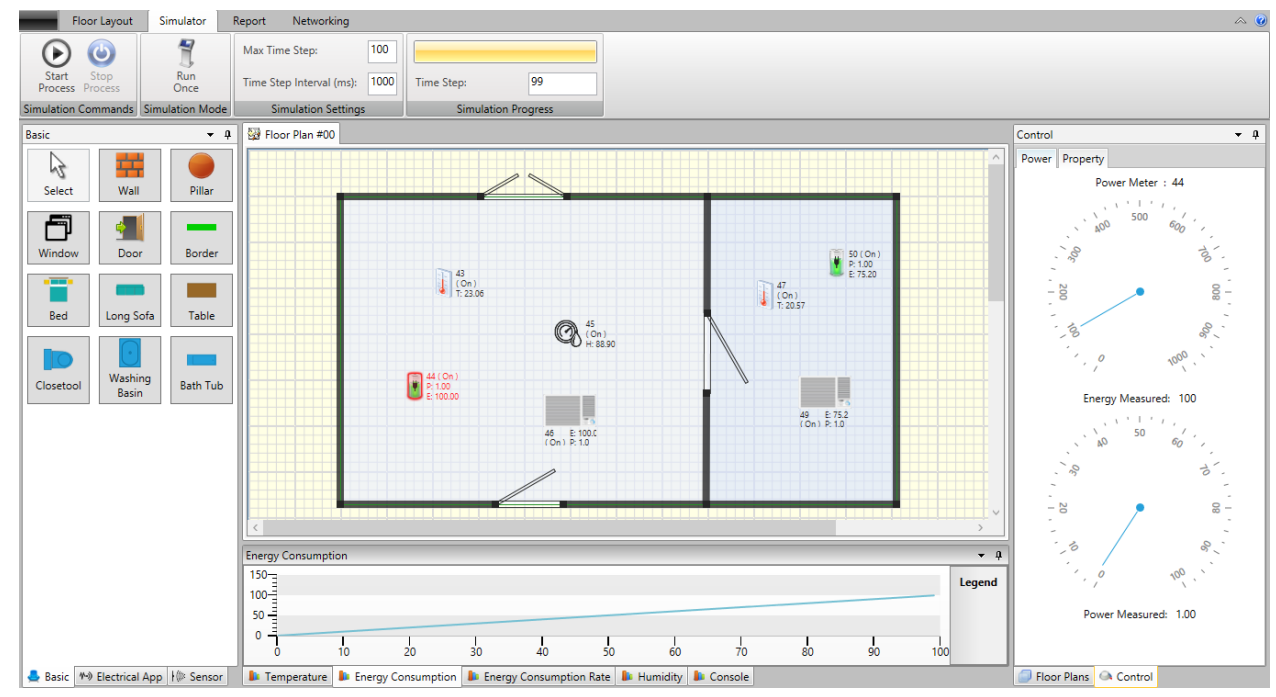

Figure 10 Optimised layout showing power consumption using the proposed algorithm (see online version for colours)

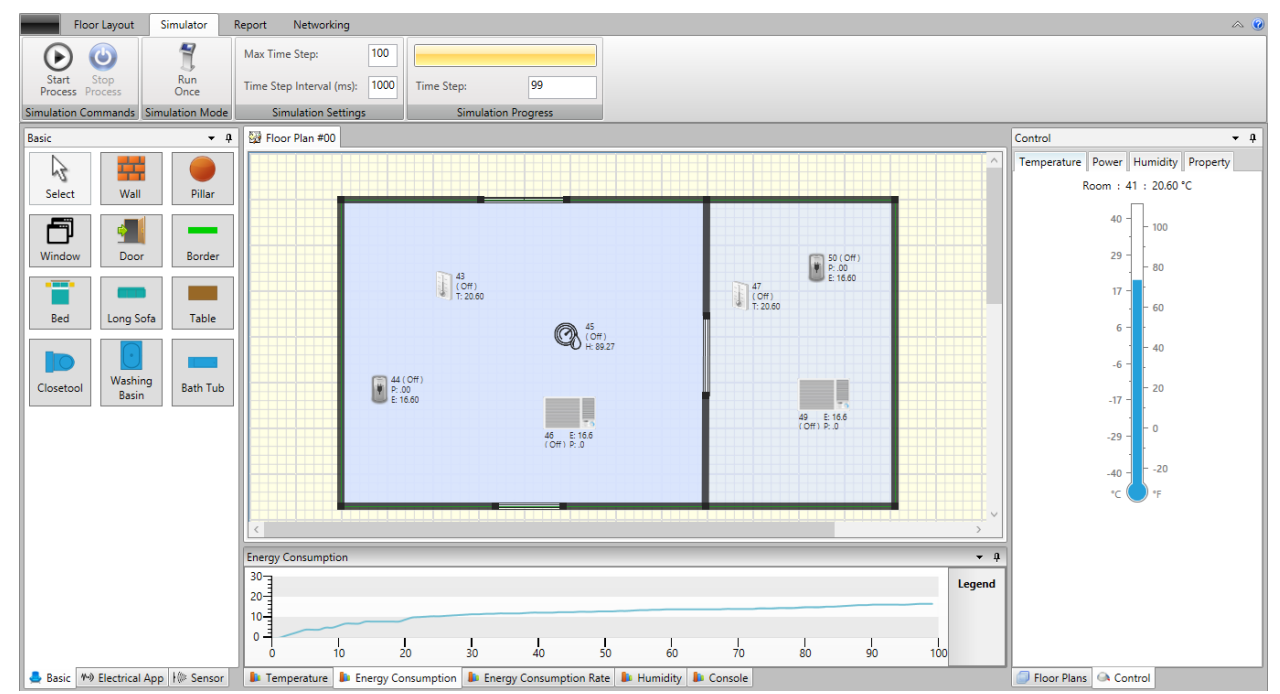


Figure 11 Optimised layout showing power consumption using HAPMOEA (see online version for colours)

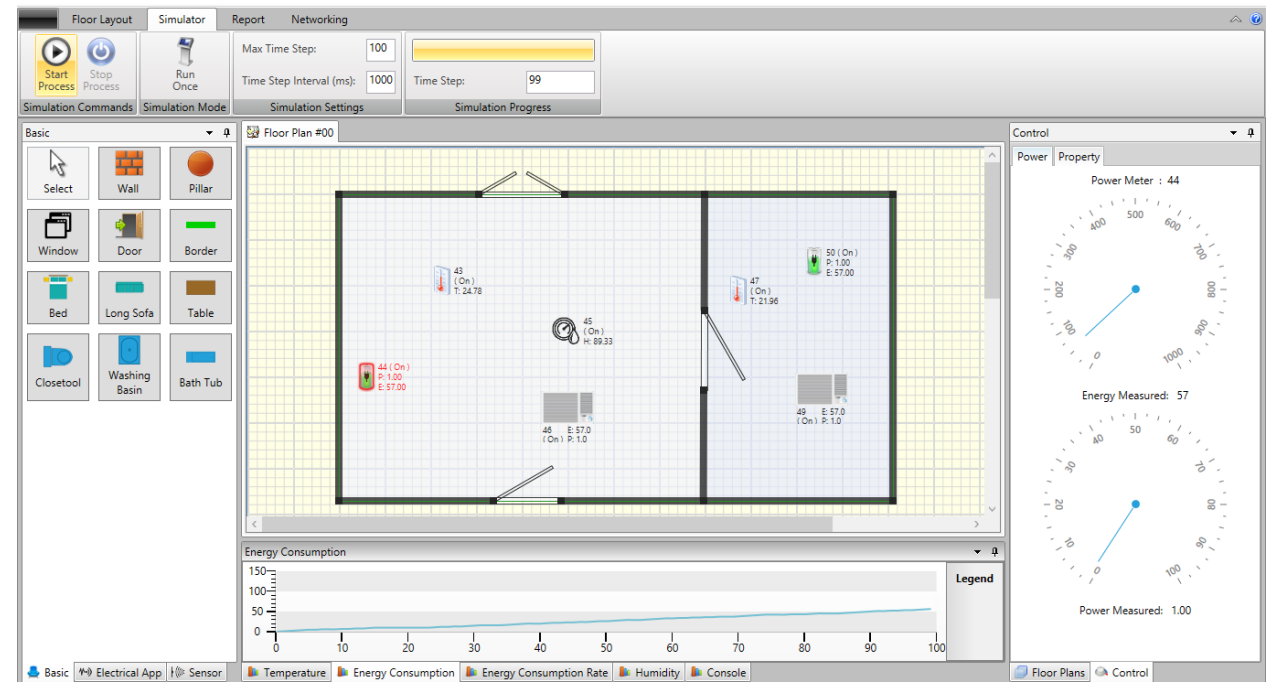

\section{Conclusions}

A new multi-objective optimisation approach is presented for generating Pareto-optimal solutions for building energy management. This method is developed by integrating Pareto dominance principles. In addition, an efficient evaluation strategy and a sequence of potential candidates with variable size are introduced. The proposed approach is applied to optimise HVAC processes by minimising power consumption without compromising the occupant comfort. Using the proposed approach, the solutions yield a trade-off among the criteria identifying a set of alternatives that define optimal solutions to the multi-objective problem. The results obtained show that the proposed approach is a viable alternative for intelligent building energy control that makes buildings more intelligent, resource efficient and comfortable for occupants. Further enhancements based on other computational intelligence approaches (Meuth et al., 2009; Gwee and Lim, 1996, 1999; Cao et al., 2006; Lim and Takefuji, 1990; Lim et al., 2002, 2004) can also be considered in future.

\section{Acknowledgements}

The authors gratefully acknowledge the funding provided by MOE Innovation Fund, Singapore. 


\section{References}

Ali, S. and Kim, D. (2013) 'Energy conservation and comfort management in building environment', Int. J. Innov. Comput. Inf. Control, Vol.9, No. 6, pp.2229-2244.

Cao, Q., Lim, M.H., Li, J.H., Ong, Y.S. and Ng, W.L. (2006) 'A context switchable fuzzy inference chip', IEEE Trans. on Fuzzy Systems, August, Vol. 14, No. 4, pp.552-567.

Dounis, A.I. and Caraiscos, C. (2009) 'Advanced control systems engineering for energy and comfort management in a building environment - a review', Renewable Sustainable Energy Rev., August-September, Vol. 13, Nos. 6-7, pp.1246-1261.

Fong, K., Hanby, V. and Chow, T. (2009) 'System optimization for HVAC energy management using the robust evolutionary algorithm', Appl. Therm. Eng., Vol. 29, Nos. 11-12, pp.2327-2334.

Giagkiozis, I., Purshouse, R.C. and Fleming, P.J. (2013) 'An overview of population-based algorithms for multi-objective optimization', Int. J. Syst. Sci., Vol. 46, No. 9, pp.1-28.

Gwee, B.H. and Lim, M.H. (1996) 'Polynominoes tiling by a genetic algorithm', Computational Optimization and Applications Journal, November, Vol. 6, No. 3, pp.273-291.

Gwee, B.H. and Lim, M.H. (1999) 'A GA with heuristic-based decoder for IC floorplanning', Integration the VLSI Journal, Vol. 28, No. 2, pp.157-172, Elsevier Science.

Kolokotsa, D., Kalaitzakis, G.S., Stavrakakis, K. and Agoris, D. (2002) 'Genetic algorithms optimized fuzzy controller for the indoor environmental management in buildings implemented using PLC and local operating networks', Engineering Applications of Artificial Intelligence, Vol. 15, No. 5, pp.417-428.

Lim, M.H. and Takefuji, Y. (1990) 'Implementing fuzzy rule-based systems on silicon chips', IEEE Expert, February, Vol. 5, No. 1, pp.31-45.

Lim, M.H., Cao, Q. and Li, J.H. (2004) 'Evolvable hardware using context switchable fuzzy inference processor', IEE Proceedings - Computer and Digital Techniques, July, Vol. 151, No. 4.

Lim, M.H., Yu, Y. and Omatu, S. (2002) 'Extensive testing of a hybrid genetic algorithm for solving quadratic assignment problems', Computational Optimization and Applications, Vol. 23, No. 1, pp.47-64, Kluwer Academic Publishers.

Meuth, R., Lim, M.H., Ong, Y.S. and Wunsch, D.C. (2009) 'A proposition on memes and meta-memes in computing for higher-order learning', Memetic Computing, April, Vol. 1, No. 2, pp.85-100.

Pervez, P.H., Mohd, N.N., Nallagownden, P. and Elamvazuthi, I. (2013) 'Intelligent optimized control system for energy and comfort management in efficient and sustainable buildings', Procedia Technol., Vol. 11, pp.99-106. 